\title{
Hard Coating is Because of Oppositely Worked Force-Energy Behaviors of Atoms
}

\section{Mubarak Ali ${ }^{\text {a }}$, Esah Hamzah ${ }^{b}$, Mohd Radzi Mohd Toff $^{\mathrm{c}}$}

a Department of Physics, COMSATS University Islamabad, Park Road, Islamabad45550, PAKISTAN, "E-mail: mubarak74@comsats.edu.pk, mubarak74@mail.com

b Department of Materials, Manufacturing and Industrial Engineering, Faculty of Mechanical Engineering, Universiti Teknologi Malaysia, 81310 Skudai, Johor, MALAYSIA, E-mail: esah@fkm.utm.my

c Advanced Materials Research Center (AMREC), SIRIM Berhad, Lot 34, Jalan Hi-Tech 2/3, Kulim Hi-Tech Park, 09000 Kulim, Kedah, MALAYSIA, E-mail: mradzit@sirim.my

Abstract -Coatings of suitable materials having thickness of few atoms to several microns on the viable substrates are the basic need of society and they attend the regular attention of scientific community working in various fields of science and technology. Decorative and protective coatings, transparent and insulating coatings, coatings of medical implants and surgical instruments, coatings for drug delivery and security purposes, ultra-precision machine coatings and coatings of miscellaneous uses are in the routine demand of research and commercial objectives. Different hard coatings develop with significant composition of differently natured atoms where their force-energy behaviors under recovering of certain transition state provide the provision for electrons (of outer ring) belonging to gas atoms to undertake another clamp of energy knot, in each case, clamping to unfilled states (of outer ring) belonging to solid atoms. Set process conditions switch force-energy behaviors of differently natured atoms as per at the ground surface where they nearly worked oppositely to the original state behaviors. Different natured atoms develop structure in the form of hard coating by locating the ground point between original points where gas atoms increase potential 
energy under the decreasing levitational force exerting at electron levels while the solid atoms decrease potential energy under the decreasing gravitational force exerting at electron levels. Ti-atom to Ti-atom binding is through the difference of expansion of their lattices when one atom is just landing on the landed atom where the position of nitrogen atoms (dealing double clamping of their electrons) becomes nearly in their interstitial sites. Under suitable set parameters, different nature atoms deposit in the form of coating at substrate surface positioned in the deposition chamber. In random arc-based vapor deposition system, depositing different nature atoms at substrate surface depends on the input power where involved non-conserved energies engaged the nonconservative forces to keep them adhered. Different properties and characteristics of hard coatings emerged as per engaged forces under the set conditions of involved energy. The present study sets new trends not only in the field of films and coatings but also in the diversified class of materials, wherever, atoms recall their roles.

Keywords: Atomic behavior; Hard coating; Expansion and contraction; Force-energy behaviors; Surface and interface

\section{Introduction}

Hard coatings are the integral part of scientific research for researching and technological advances. Marketable hard coatings for different purposes are learnt routinely where their ingredients and deposition technique are in the hot topics. In this context, several materials involving different ingredients and deposition techniques are available in the literature discussing and explaining their deposition history along with the features of substrate material. In coatings, a minute deposited quantity of materials over less-important or not practically viable material solves the purpose of giving the value-added results.

A variety of techniques are involved to deposit different sorts of hard coatings at the surface of suitable substrates. Coatings are mainly employed with two targeted approaches; in the first case, the potential use of external coated part, which is not subjected to a great deal in the uncoated case and, in the second case, potential use of internal surface where inner uncoated surface of material was not the subject of great 
concern but, on coating, it became a great deal. Overall, coating the surface of a certain substrate results into deliver its different behavior of functioning, often in an astonishing way.

Solid atoms of unfilled states do not elongate and those belonging to inert gases split under the excess propagation of photons characteristic current [1]. A neutral state silicon atom transformed heat energy into photon energy as discussed elsewhere [2]. Atoms of suitable solid behavior evolve structures of different dimension and format as per the nature of built-in electronic gauge where conservative forces involved to execute confined inter-state electron-dynamics [3]. The origins of atoms of certain elements to be in gas state while the origins of atoms of certain elements to be in solid state are discussed elsewhere [4]. A gas state carbon atom originates several different physical behaviors, which is under the involvement of non-conserved energy where confined inter-state dynamics of electron to attain its certain state engaged non-conserved forces as well [5]. Depending on the atomic behavior of tiny sized particles, their role for nanomedicine can either be beneficial or harmful [6].

Developing different hierarchical tiny particles under varying conditions of the process is under differently attained dynamics of gold atoms [7]. A monolayer triangularshaped tiny particle was considered as the model system discussing the elongation behavior of one-dimensional arrays of gold atoms and their conversion into structure of smooth elements under the joint application of surface format force and travelling photons of adequate wavelength [8]. At suitable precursor concentration, many tiny particles shape-like equilateral triangle developed as discussed elsewhere [9]. Shapes of tiny sized particles and large sized particles were controlled under the application of different ratios of pulse OFF to ON time [10]. Particles of different anisotropic shapes developed in less than millisecond time [11]. Developing tiny sized particles of certain shape tapped in different precursors [12]. Predictor packing while developing highly geometric anisotropic gold particles is discussed elsewhere [13] where controlled forceenergy behaviors regulate the shape.

Different behaviors of tiny grains carbon films registered under Raman spectroscopy and energy loss spectroscopy indicated several phases of tiny grains [14]. Switching Last and Final Version 
morphology-structure of grains and crystallites under a bit altered locally operating parameters in developing carbon films is discussed elsewhere [15]. Under varying chamber pressure, a discernible change in the morphology and growth rate of carbon films was observed [16].

While depositing TiN coatings on different substrates under varying process conditions while employing a technique known as 'cathodic arc physical vapor deposition', a different morphology-structure along with hardness, surface roughness, friction coefficient, adhesion strength and overall performance of coated tools were studied [17-28]. In addition to these studies, there are several other available studies in the literature targeting TiN coatings along with their processing techniques and analyses [29-36]. In addition to TiN coating, different types of hard coatings developed under various employed conditions have also been published extensively [37-48]. The basic idea discussed in those studies is related to the properties and characteristic of deposited coatings, which are subjected mainly to the change of process parameters, type of material and processing approach.

The prosperous assembling of colloidal matter into meaningful structure will result into deal atoms and molecules as tomorrow's materials [49] and understanding in the individual dynamics of tiny sized particles formation is essential before enabling their assembling to the useful large sized particles [50].

In addition to the discussed scientific details available for hard coatings, coatings are in the way to express relation between comprised atoms. This study reports the fundamental aspects of developing hard coatings with special emphasis on depositing TiN coating at a high-speed steel (HSS) disc while employing random arc-based vapor deposition technique. This work presents the fundamental aspects of depositing hard coating, in general, and investigating mechanism of developing TiN coating, in specific.

\section{Experimental details}

HSS discs were utilized as a substrate material for the deposition of TiN while employing the commercially available coating unit known as 'cathodic arc physical vapor deposition', which is now termed as 'random arc-based vapor deposition' in the Last and Final Version 
present study. After the required cleaning, the samples having diameter: $10 \mathrm{~mm}$ and thickness: $6 \mathrm{~mm}$ were loaded in the coating system mark Hauzer Techno Coating (HTC) $625 / 2$ ARC. The complete deposition procedure along with metallographic process of samples have been described in our earlier work [28]. Surface and interface studies were done using FE-SEM (Model LEO-1525). The thickness of the deposited coatings was measured by using the application of FE-SEM under the fractured cross-sectional image. Prior to go for coating TiN, an interlayer of Ti was deposited for 15 minutes time to enhance the adhesion strength of the following coating. At start of depositing interlayer, chamber pressure was $5 \times 10^{-6}$ mbar. While depositing inter-layer, $50 \mathrm{sccm}$ nitrogen gas flow rate was maintained by mass flow controller meter. To deposit TiN in the form of coating, substrate temperature was maintained at $300^{\circ} \mathrm{C}$ where $\mathrm{N}$ gas flow rate was $250 \mathrm{sccm}$. The bias voltage was 50 volts where rotational speed of the substrate holder was set $60 \%$. Current for igniting arc to eject Ti-atoms from their target was $100 \mathrm{~A}$. Total duration of the deposition process was set 90 minutes.

\section{Results and discussion}

Figure 1 (a) shows surface morphology of deposited TiN on HSS disc where coating is partially covered with macrodroplets (MDs), size ranging from few hundred of nanometers to few microns. The distribution of MDs is uniform throughout the surface. A large sized macrodroplet (MD) in the central vicinity shows mapping of the region where the concentration of both $\mathrm{Ti}$ and $\mathrm{N}$ atoms is appeared in different colors. Figure 1 (b) shows fractured cross-sectional view of the coating where initially deposited $\mathrm{Ti}$ layer shows thickness less than one micron. Atoms of Ti-interlayer first adhere to surface atoms of substrate under the favorable conditions.

Substrate surface comprises different elements like $\mathrm{W}, \mathrm{Mo}, \mathrm{Cr}, \mathrm{V}, \mathrm{C}$ and $\mathrm{Fe}$, which facilitate the initial adherence of Ti-atoms to the substrate [17, 24]. Ti-atoms bound to substrate surface for introducing the favorable conditions of depositing TiN coating. In this context, the nature of substrate can possess different elements where their certain force-energy behaviors introduce appreciable binding to the depositing atoms. Adhesion

\section{Last and Final Version}


strength of TiN coating deposited under certain conditions in random arc-based vapor deposition system is discussed elsewhere [26, 28].
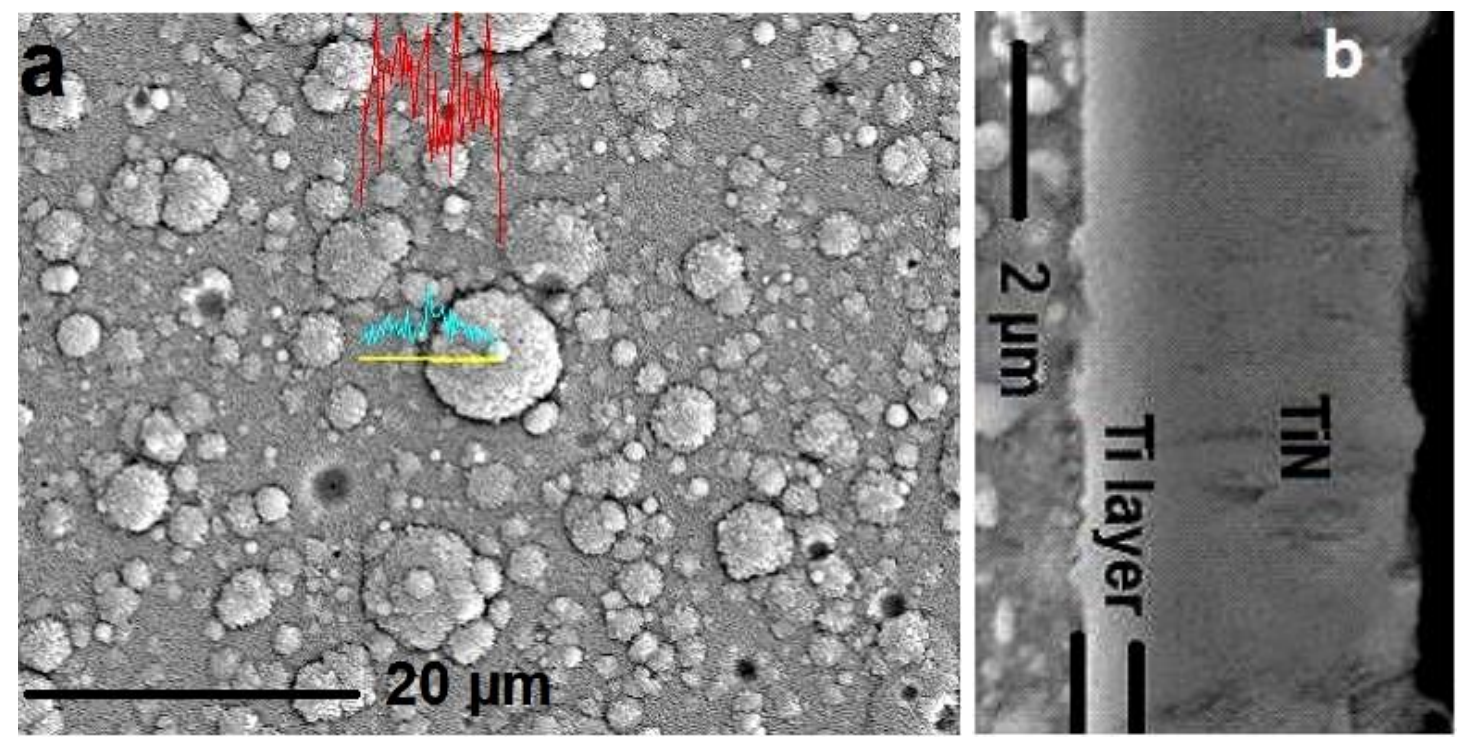

Figure 1: (a) topographic view of TiN coating on HSS and (b) titanium interlayer in few hundred nanometers shows contrast to TiN coating deposited in thickness of $\sim 4 \mu \mathrm{m}$ (a cross-sectional view of coating prepared by metallographic cross-sectioning procedure)

Figure 2 shows the mapping of Ti-atoms present in TiN coating at the point of MD (shown in Figure 1a) in the form of histogram where the content is around $70 \%$. This indicates that the area covered by Ti-atoms in the TiN coating at top front surface not only contained $70 \%$ of the content but the distribution of atoms in MD also remained dense and uniform. This indicates that MD contained less concentration of $\mathrm{N}$-atoms. 


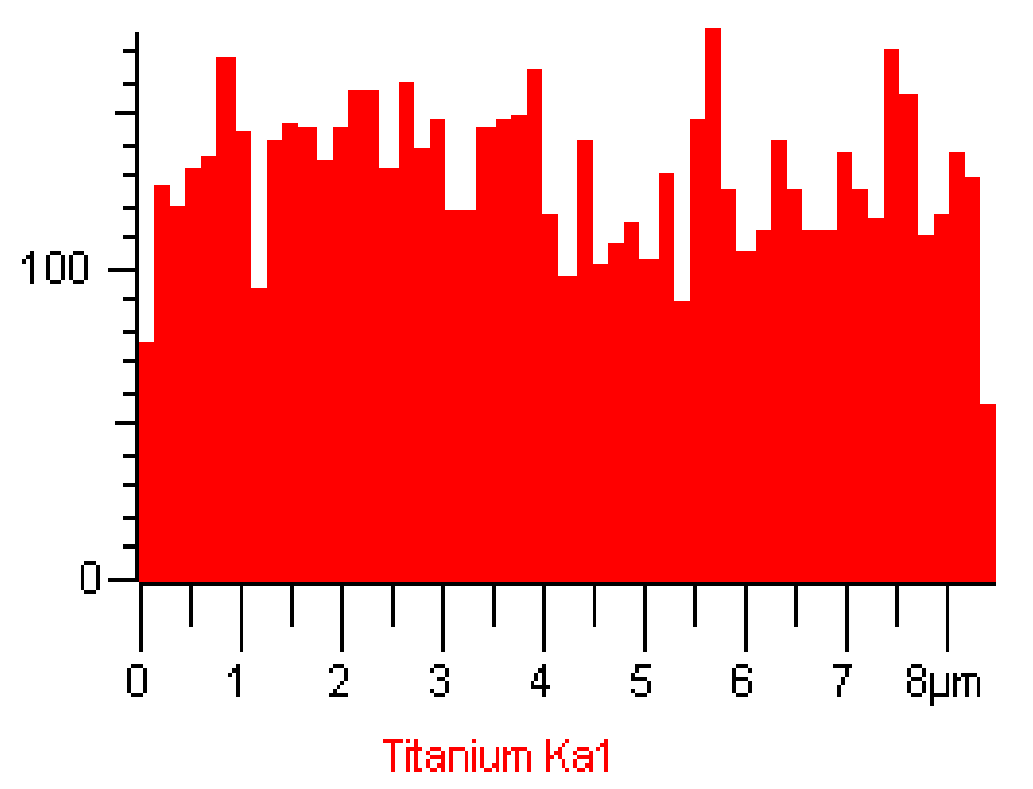

Figure 2: Mapping of Ti-atoms distribution along with the ratio of content

Figure 3 shows the mapping of $\mathrm{N}$ content in TiN coating in the form of histogram, which is around $30 \%$ at the central point of MD shown in Figure 1 (a). This indicates that the area covered by $\mathrm{N}$-atoms in the TiN coating at top front surface not only contained $30 \%$ of content but also contained uniform distribution, however, not in a dense manner. This describes that MD contained approximately three times less concentration of $\mathrm{N}$ atoms as compared to Ti-atoms.

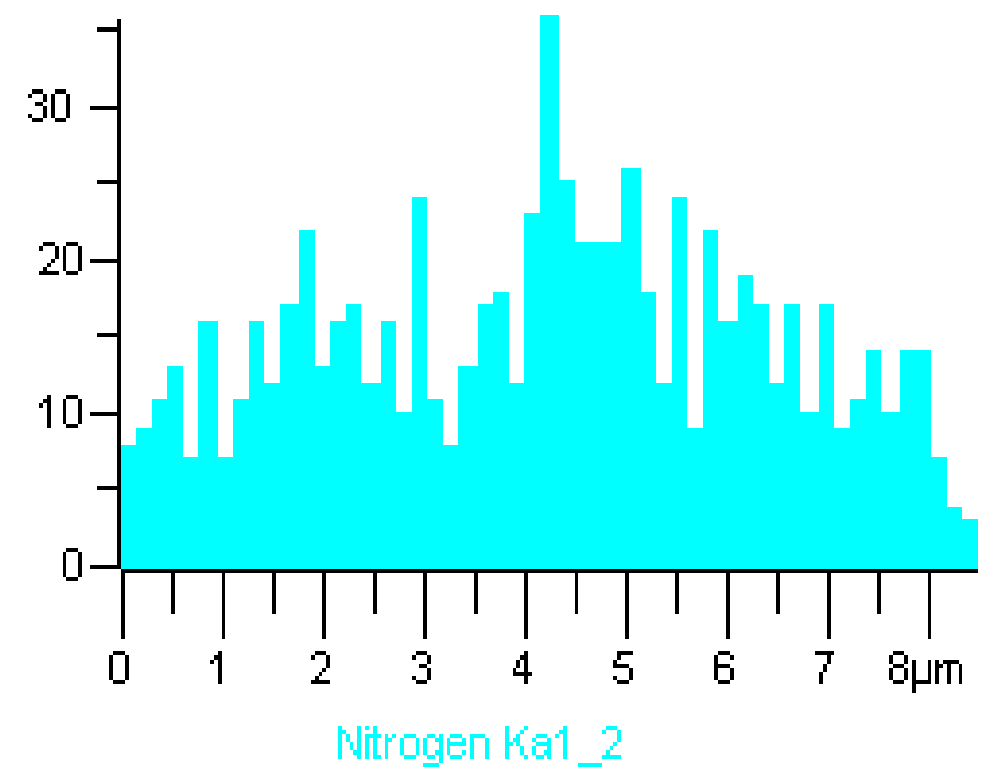

Figure 3: Mapping of $\mathrm{N}$-atoms distribution along with the ratio of content

\section{Last and Final Version}


There are several studies studying the reduction of MDs under varying the process parameters in vapor deposition technique along with different employed strategies [1720, 23-25].

Hard coatings mainly fall in the category of refractory materials and they are no more the candidates of conductive behavior despite of the fact that major component of those coatings still involve metallic nature atoms. On adhering gas atoms to metallic atoms, the resulted coatings become poor in conduction and they work nearly as an insulator where field of propagating photons characteristic current is interrupted to a large extent. This is because of locking inter-state electron gaps for metallic nature atoms by means of incorporating gas atoms. These result into develop disordered structure of the resulted coating. In the case of disordered structure, it is only within the short-range order. The incorporated $\mathrm{N}$-atoms built the bridges via their certain electrons where they undertake another clamp of energy knot (for electron of each atom) clamping to unfilled state of certain Ti-atom. This resulted into lower the propagation of photonic current. A detailed study is given elsewhere [1] discussing the significance of inter-state electron gaps in atoms of different elements; propagation of current no more requires the band gaps between conduction bands and valence bands as they are not reliable for explaining the science of semiconductor materials or other types of materials.

In random arc-based vapor deposition system, atoms of $\mathrm{Ti}$ (or other metallic nature atoms) are ejected from the exposed surface of targets where arc is utilized to eject atoms under the supply of energy (a bit in non-uniform manner) depending on the strength of applied field per unit area (or volume). At high concentration of $\mathrm{N}$-atoms, a random arc is steered to eject Ti-atoms both in atomic form and tiny-sized cluster (droplet) depending on the nature of Ti targets along with employed conditions of vapor deposition process. The basic layout of ejecting Ti-atoms and entering $\mathrm{N}$-atoms to deposit TiN coating is sketched in estimation, which is shown in Figure 4. 


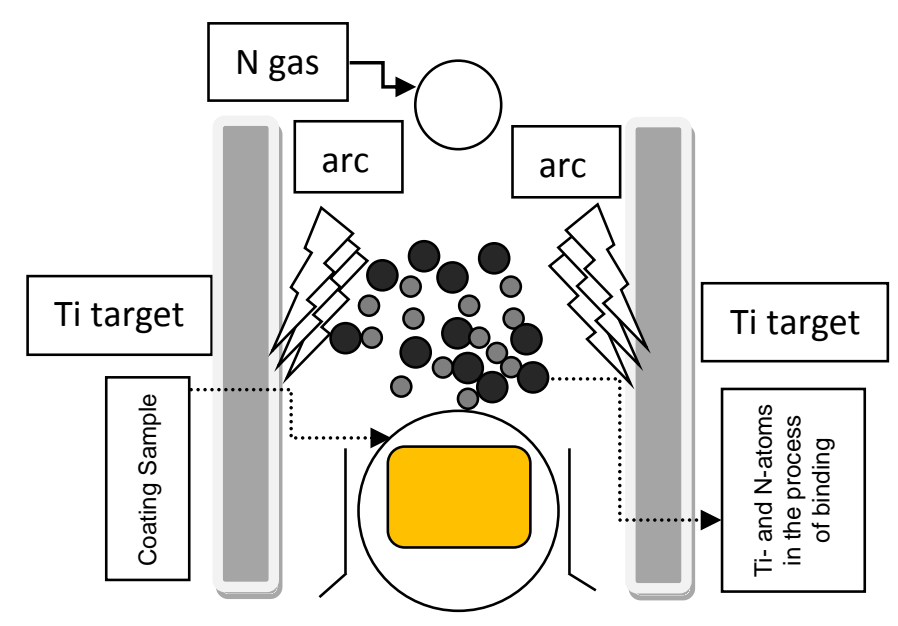

Figure 4: The basic layout of depositing Ti-atoms and $\mathrm{N}$-atoms to develop TiN coating at the surface of HSS substrate

Expected atomic structure of $\mathrm{Ti}$ and $\mathrm{N}$ is shown in Figure 5. The tinniest sized particles called electrons are filled in the hollow space constructed by the inter-crossed overt photons in certain symmetry having wavelength in photons characteristic current. In the case where electrons don't fill those hollow space, they are termed as the unfilled states of atoms. For $\mathrm{Ti}$ atom, total 32 states of electrons are available, but 24 states are filled by the tinniest sized particles (called electrons) while 8 states are remained unfilled. In the case of inner unfilled states of the atom, they are being remained pressed by the covered filled states as indicated in Figure 5 . Both filled and unfilled states of $\mathrm{Ti}$ atom are constructed by the inter-crossed overt photons having their dedicated length. The required number of overt photons are being inter-crossed with full understanding of their atoms related to each element by involving the three-dimensional space. However, unfilled states are belonged to outer ring where electrons don't occupy the position, which are at the terminals of certain chain of states as shown in Figure 5. 


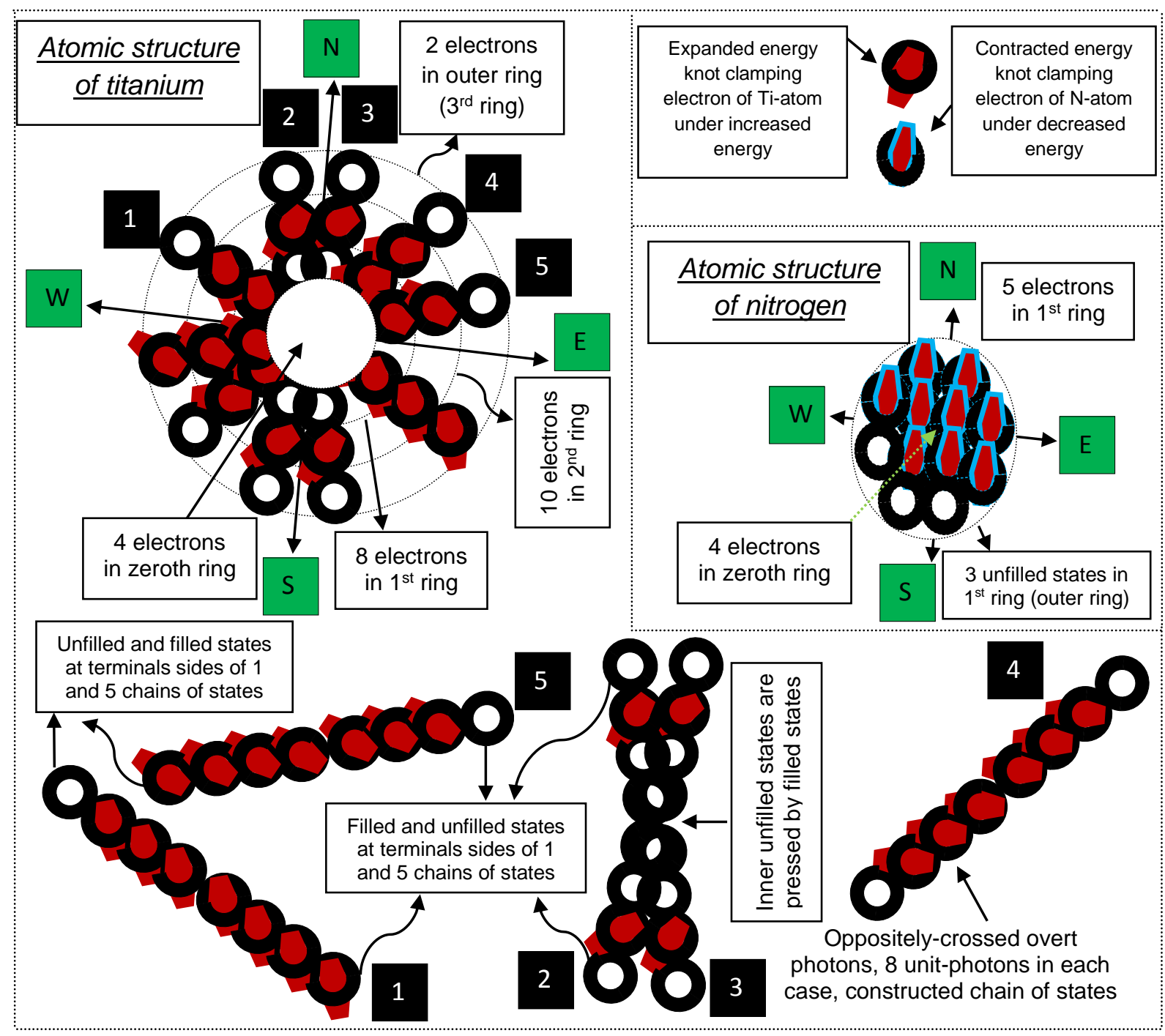

Figure 5: Expected atomic structure of Ti and $\mathrm{N}$; involved different chain of states are shown at bottom indicating filled, unfilled states and pressed states for Ti-atom

Oppositely-sided crossed two overt photons comprising eight unit-photons construct chain of filled and unfilled states of electrons. As shown in the bottom part of Figure 5 where five such shapes are drawn and their precise inter-crossing at a common centre form the lattice of Ti-atom in which 24 states are remained filled. Expanded and contracted energy knots (in estimation) clamping electrons in Ti-atom and $\mathrm{N}$-atom, respectively, are also shown in Figure 5. Filled states of outer ring in the atoms of solid and gas behaviors donate the positive valency and negative valency, respectively. For the case of Ti atom, valency is +2 , so, it has ' 8 ' unfilled states. In the case of nitrogen Last and Final Version 
atom, it has valency -3 , so, it has ' 5 ' unfilled states. Hence, negative sign for valency in gas behavior atoms indicates their ground point at above average level of ground surface and positive sign for valency in solid behavior atoms indicates their ground points at below average level of ground surface. Inner four electrons of atoms belonging to each element form the zeroth ring, whereas, others are related to the available consecutive rings as discussed elsewhere [4].

In different coating technology units, regardless of that, required numbers of atoms per unit area or volume are being deposited under set parameters of the process, their involved energy is based on individually attained dynamics plus electron-dynamics, which is the key to regulate their structure, so, there is emergence of properties and characteristics of their resulted coating. However, it appears that evolving structure of TiN coating in the order of certain homogeneity is within the short-range order. Therefore, the deposited coating is developed mainly under the mixed behavior of structure evolution. Each Ti-atom only occupies two electrons in the outer ring. This low number of its filled states enables it to occupy many unfilled states of outer ring since its existence. Ti being a solid atom is supposed to has its unfilled states at above east-west poles, both at left and right sides of the north-pole. Because atoms of Ti element are belonged to grounded format where electrons of filled states (in the outer ring) are to be remained at below their east-west poles along the south-pole while dealing their original solid behavior. On the other hand, five filled states in outer ring of $\mathrm{N}$-atom allow a smaller number of unfilled states in the outer ring. In this context, a $\mathrm{N}$-atom contains several filled states of outer ring where majority of the electrons (filled states) are expected to be at above the east-west poles along the north-pole while in each dedicated state. This is because of the gas nature of $\mathrm{N}$-atom. Further detail of the origin of gas atoms belonging to some elements was discussed [4]. The availability of several unfilled states of outer ring in Ti-atom provide provision to work for electrons of filled states of outer ring in $\mathrm{N}$-atom where targeted electron (of gas atom) is being clamped by another clamping of energy knot (of solid atom). The double-clamping of energy knot to the electron of $\mathrm{N}$-atom is by means of energy knot clamping unfilled state of outer ring in the Ti-atom. But the mechanism of double clamping of energy knot by the electron is by 
means of suitable transition of both gas and solid atoms. Atoms when are in a certain transition state adjust potential energy of their electrons as per exertion of the orientating force [4]. Therefore, two different nature atoms ( $\mathrm{Ti}$ and $\mathrm{N}$ ) develop affinity in terms of strong binding under a suitable reaction (fast interaction).

A metallic target developed under the solidification of undertaking transition state atoms, most probably, a re-crystallization state. The processed ore of metallic target is at the level of ground surface but their atoms in original solid state are to be at the below level to ground surface. Similarly, gas atoms compressed in the container are in the re-crystallization state as well at the level of ground surface, whereas, they are in original gas state at the above level to ground surface. On ejecting of solidified natured atoms from their target and flowing of the gas natured atoms from their compressed form (in the container), they are again in transition to restore their original states. So, in the attempt of reviving their original state behaviours, they react (fast interact) just at above the substrate of their deposition. At instant of their reaction (fast interaction), different natured atoms are in their nearly opposite switched force-energy behaviours (re-crystallization state). Here, under their suitable interactions, electrons of gas atoms enter to unfilled states of solid atoms. In both cases, entering electrons of filled states and clamping energy knots of unfilled states, they are belonged to the outer ring of their atoms. Gas atoms when are in the re-crystallization state, their electrons move to downward sides under infinitesimal displacements where they decreased their levitational force under increasing the potential energy. But, electrons of the gas atoms are still more than $50 \%$ to upward sides at mid of their clamped energy knots. Solid atoms when are in the re-crystallization state, their electrons move to upward sides under infinitesimal displacements where they decreased their gravitational force under decreasing the potential energy. But, electrons of the solid atoms are still more than $50 \%$ to downward sides at mid of their clamped energy knots. So, when two different natured atoms reach to just in neutral state, electrons of the gas atoms undertake clamps of energy knots belonging to solid atoms, in each case where clamping of one more energy knot to each electron (of the gas atom) is belonged to the solid atom. Those different natured atoms restore their original state by remaining adhered where Last and Final Version 
bringing the hard features now by introducing the maximum potential energy (under the maximum expansion of energy knots of filled and unfilled states) of bound gas atoms also. When gas and solid atoms are just in neutral states, electron of the gas atom undertake one more clamping of energy knot belonging to solid atom by visualizing the exerting forces of its relevant poles.

Atoms of metallic targets are already in the contraction of energy knots clamping electrons because, now they are not grounded below the ground surface. On the other hand, entered gas atoms to the chamber are in the expansion of energy knots clamping electrons because, now they are at ground surface instead of being at above ground surface through the given pressure for their entering to the deposition chamber. Therefore, different nature atoms switched force-energy behaviors nearly in opposite manner. Just at instant of recovering original behaviors, they reach to bind upon suitable coinciding where targeted electron of gas atom undertakes another clamp of targeted energy knot (clamping to unfilled state) of solid atom. Therefore, solid atoms of Ti have already done work negatively (arriving near to ground surface from the southside) while gas atoms of $\mathrm{N}$ have already done work positively (arriving near to ground surface from the north-side). On recovering the state behaviors of two differently natured atoms to be just in neutral state from their re-crystallization states they switched their force-energy behaviors where work done by the gas atom is negative while work done by the solid atom is positive. So, they react to undertake double clamping of the suitable electron (of the gas atom) through suitable unfilled state (of the solid atom).

Ti metal is known in metallic character where filled state electrons keep their atoms grounded under the maximum gravitational force and the maximum expansion of their clamped energy knots, also. Thus, electrons of Ti-atoms keep original ground point at below ground surface under the normal condition. On the other hand, a $\mathrm{N}$-atom belongs to gas behavior, which remains in the air under the engagement of space format force exerting at electron levels, thus, its electrons keep original ground point at above ground surface. The electrons of $\mathrm{N}$-atoms keep their ground point at above ground surface because of the maximum contraction of their clamped energy knots where the maximum orientating levitational force is being exerted at electron levels. Therefore, in Last and Final Version 
their joint deposition while employing a suitable coating technology unit, electron of outer ring belonging to $\mathrm{N}$-atom clamp by another energy knot clamping to unfilled state of outer ring in Ti-atom. Given energy in the deposition chamber enables another clamping of the energy knot to suitable electron of gas atom and through energy knot of suitable unfilled state of solid atom where energy is involved to engage the force. This results into search their ground point neither at above ground surface nor at below ground surface. In the search of attaining their ground points, they mutually get ready for the common ground point at the surface of depositing substrate, which becomes nearly at mid-point of two different ground points; at above ground surface (in gas atoms) and at below ground surface (in solid atoms). Under the tailored process parameters of deposition, structure of hard coating deal high hardness because of the maximum ordering of different nature transition state atoms where their attained midpoints remained ordered to a large extent.

The electrons of $\mathrm{N}$-atoms undertake double clamps of energy knots while focusing to unfilled states of Ti-atoms where gas state atoms are being uplifted while solid ones are being grounded. Suitable transition state atoms undertake double clamps of targeted energy knots (belonging to solid atoms) to targeted electrons (belonging to gas atoms) under their favorable coinciding. The mechanism of double clamping of suitable energy knots to suitable electrons of $\mathrm{N}$-atoms ground forcefully by the Ti-atoms (through suitable energy knots clamping unfilled states) as shown in Figure 6. Binding of Ti-atom to Ti-atom under the application of an electron (belonging to less expanded Ti-atom just landing) to undertake another (double) clamp of energy knot (belonging to more expanded Ti-atom already landed) is also shown in Figure 6 where $\mathrm{N}$-atoms mainly position at interstitial sites of Ti-atoms. Under the action of tailored force-energy behaviors of $\mathrm{N}$-atoms and Ti-atoms, they react, which results into their binding to develop TiN coating at surface of the substrate. Because, solid atoms in their original state behavior dealt fully orientated gravitational force, which is due to their grounded ground points under fully gravitized behavior exerting at electron levels, thus, their electrons gained the maximum potential energy where clamped energy knots also dealt the maximum expansion. So, energy knots constructing unfilled states in those atoms Last and Final Version 
also expanded maximally. But, gas atoms in their original state behavior dealt fully orientated levitational force, which is due to their ground points at above ground surface under fully levitized behavior exerting at electron levels, thus, their electrons gained the minimum potential energy where clamped energy knots also dealt the maximum contraction. So, energy knots constructing unfilled states in those atoms also contracted maximally.

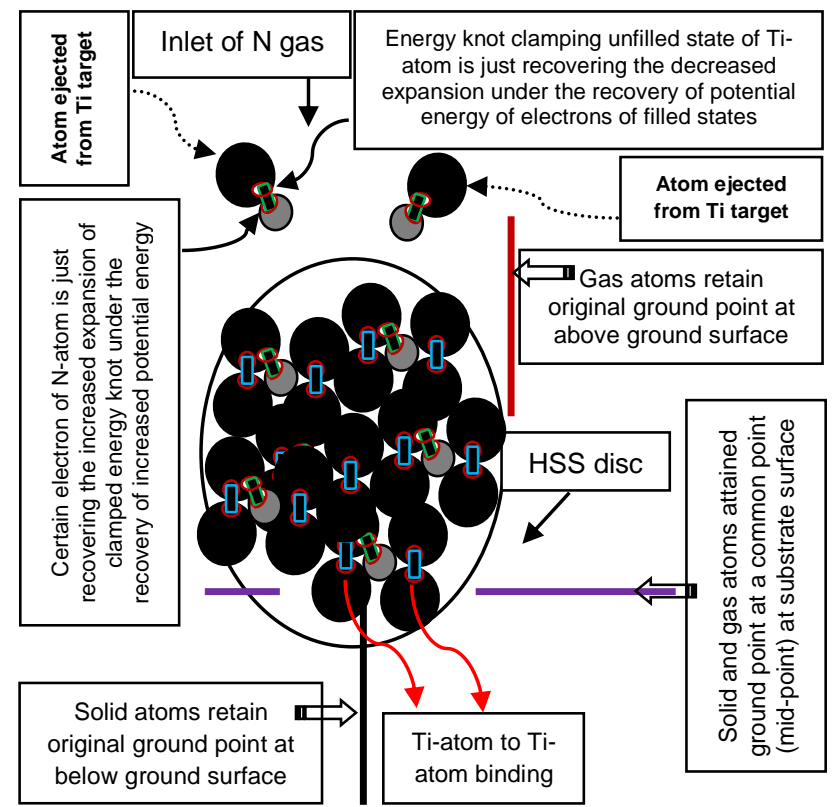

Figure 6: Mechanism of double clamping of suitable energy knots to suitable electrons of gas atoms through suitable energy knots clamping unfilled states of solid atoms along with Ti-atom to Ti-atom binding

On landing a Ti-atom at substrate, it recovers its transition state into original solid behavior where electrons start to gravitize. But prior to fully gravitize, an adequate expansion of the lattice under increased (gained) potential energy is taken place. However, Ti-atom after landing attains ground point at the surface of previously landed atom, so, it kept the lattice in less expansion. Therefore, a certain electron of less expanded land Ti-atom (where it is pointing toward the downward side) enters to a suitable vacant energy knot of a more expanded landed Ti-atom (where it is pointing toward the upward side). This results into bind the two identical atoms. Now, forcefully grounded nitrogen atoms tend to recover state to go into original gas behavior where 
their certain electrons are entered to certain vacant energy knots of Ti-atoms from the back-side. In this way, nitrogen atoms are trapped at interstitial position of the Ti-atoms. Binding of nitrogen atoms at interstitial positions of bound Ti-atoms is also shown in Figure 6. Atoms of nitrogen (or other suitable gas elements) and titanium (or other suitable solid elements) engage energies under the exerting forces of fixed poles of electrons when they in their original behaviors. But, the situation becomes different when they undertake certain transition (liquid) state where in the solid atoms, energy is directly proportional to the exerting gravitational force exerting at electron levels, whereas, in the gas atoms, energy is inversely proportional to the levitational force exerting at electron levels.

The similar sort of mechanism is being anticipated in bi-metallic composition with gas behavior atoms, for example, TiAIN. Again, low measured-hardness coating of chromium nitride (compared to TiN) involves the mechanism of binding their different nature atoms under similar lines where high probability of binding is involved as $\mathrm{Cr}$-atom contains many unfilled states in the outer ring (compared to Ti-atom). That's why CrN coating exhibits low surface roughness as compared to TiN coating [28] and, also, because of greater level of homogeneity of binding atoms while evolving structure. A similar approach may be validated to explore the science of other hard, moderate hard and even less hard (soft and porous) coating materials. A different originating scientific mechanism may be anticipated in the case of TiCN coating and because of involving the carbon atoms, which requires additional lines to express the science of their synthesizing materials. Reacting of gas atoms and solid atoms endorse engaging of force exerting by their electrons under the adjustment of expansion and contraction of clamped energy knots, respectively. In this case, the energy is being involved. Therefore, developing hard coating is related to non-conserved energy where nonconservative frictional forces are engaged to sustain the structure of coating. However, where the force element is involved first, the energy is being engaged as for the case of silicon atoms [2]; conservative forces are involved to configure the energy in the form of forcing energy (photon). A photon wavelength in the characteristic current is discussed elsewhere [1]. A unit-photon and an overt photon are discussed elsewhere [2]. The Last and Final Version 
origin of atoms of different elements to be in gas state and to be in solid atoms is discussed elsewhere [4]. Conversion of gas state carbon atom into different states carbon atoms along with the binding of identical state carbon atoms is also discussed [5].

\section{Conclusions}

This is the provision of solid atom just recovering the transition state which allocates energy knot belonging to suitable unfilled state of outer ring to clamp certain filled state electron of gas atom when just recovering the transition state. The reacting of gas atom while ground point is just at ground surface (instead of at above ground surface) is because of increased orientating gravitational force where potential energy of electrons is increased under the increased expansion of the lattice. The reacting of metallic atom while the ground point is just at ground surface (instead of at below ground surface) is because of decreased orientating gravitational force where potential energy of electrons is decreased under the decreased expansion of the lattice. This results into the binding of two different natured atoms at a common ground point, which is the mid of ground points of gas atom and solid atom when they are in their original state behavior, thus, engage force as per involved energy to sustain binding working as a hard coating.

To be undertaken by the electron (belonging to filled state of gas atom in its outer ring) one more clamping of energy knot (belonging to unfilled state of solid atom in its outer ring) is through the mutual adjusting expansion-contraction of their lattices resulting into deposit structure of hard coating, which is known since antiquity.

The underlying science of developing hard coating is in the manner that atoms of solid behavior perform negative work when undertaking the certain transition state as they attain ground points at the level of ground surface and atoms of gas behavior perform positive work when undertaking the certain transition state as they attain ground points at the level of ground surface. To develop hard coating, gas atoms when just recovering transition under increased orientating gravitational force expanded lattice to react solid atoms of contracted lattice when just recovering transition under decreased orientating gravitational force. Two differently natured atoms adhere to each Last and Final Version 
other when they are in the neutral transition states where electron (belonging to gas atom) when undertaking another clamp perfectly visualizes the exerting force through energy knot clamping to unfilled state (belonging to solid atom).

Transition metals govern hard features of coating under affinity to gas atoms because, one is undertaking the force of grounded format and other is undertaking the force of space format resulting into locate their common ground points having midpoints at the level of ground surface. The electron of outer ring belonging to gas atom (N-atom) reacts to develop TiN where it undertakes another clamp of energy knot clamped by the unfilled state of outer ring belonging to solid atom (Ti-atom).

At the time of recovering transition of Ti-atoms, they are just at the substrate surface, thus, they react to $\mathrm{N}$-atoms, which are also just at substrate surface at the instant of recovering transition. Just at the landing, Ti-atoms deal less expansion of energy knots clamping electrons which also undertake double clamping of energy knots to their certain electrons by means of energy knots clamping certain unfilled states of already landed Ti-atoms where extended level of expansion of their lattices occurs. This results into devise the unit (primitive) cell of hard coating under their appropriate coinciding. This is the cause that hard coating presents the increased elastic behavior and the decreased plastic behavior.

Appropriate vacuum conditions and high power enhance the hardness level of deposited coatings. Hard coatings introduce certain properties and application due to the developing of non-regular structure where non-conservative energies involve, thus, engaging the non-conservative forces to sustain their structure. But, they are not overwhelming forever where their force-energy behaviors may be affected. So, the lifetime of hard coatings depends on their developing strategies along with nature of application. Hard coatings explore new sciences of opening many areas of research at new grounds.

\section{Acknowledgements:}

Mubarak Ali is obliged to Malaysian Government for awarding scholar of $\mathrm{PhD}$ study (2004-07) under award letter No. JPA(L) KD333487 and Government of Pakistan 
(MoST) for granting the study leave. He is also grateful to Malaysian colleagues at AMREC, SIRIM Berhad and at UTM Johor for their kind hospitality. Special thanks to Professor Dr. Ali Ourdjini for reviewing the work at several times and constructive feedback while studying at UTM who is now with the University of Ottawa, Canada.

\section{References:}

[1] M. Ali, Atoms of electron transition deform or elongate but do not ionize while inert gas atoms split. http://arxiv.org/abs/1611.05392 (last version)

[2] M. Ali, Revealing the Phenomena of Heat and Photon Energy on Dealing Matter at Atomic level. https://www.preprints.org/manuscript/201701.0028/v10

[3] M. Ali, Structure evolution in atoms of solid state dealing electron transitions. http://arxiv.org/abs/1611.01255 (last version)

[4] M. Ali. Why Atoms of Some Elements are in Gas State and Some in Solid State, but Carbon Works on Either Side. https://www.researchgate.net/profile/Mubarak Ali5

[5] M. Ali, Atomic Structure and Binding of Carbon Atoms, (2018). https://www.preprints.org/manuscript/201801.0036/v5

[6] M. Ali, Nanoparticles-Photons: Effective or Defective Nanomedicine, J. Nanomed. Res. 5 (6) (2017) 00139.

[7] M. Ali, I -N. Lin, The effect of the Electronic Structure, Phase Transition, and Localized Dynamics of Atoms in the formation of Tiny Particles of Gold. http://arxiv.org/abs/1604.07144 (last version)

[8] M. Ali, The study of tiny-shaped particle dealing localized gravity at solution surface. http://arxiv.org/abs/1609.08047 (last version)

[9] M. Ali, I $-\mathrm{N}$. Lin, Development of gold particles at varying precursor concentration. http://arXiv.org/abs/1604.07508 (last version)

[10] M. Ali, I $-\mathrm{N}$. Lin, Controlling morphology-structure of particles at different pulse rate, polarity and effect of photons on structure. http://arxiv.org/abs/1605.04408 (last version) 
[11] M. Ali, I-N. Lin, Formation of tiny particles and their extended shapes - Origin of physics and chemistry of materials. http://arxiv.org/abs/1605.09123 (last version)

[12] M. Ali, I -N. Lin, C. -J. Yeh, Tapping Opportunity of Tiny-Shaped Particles and Role of Precursor in Developing Shaped Particles. NANO 13 (7) (2018) 1850073 (16 pages). https://doi.org/10.1142/S179329201850073X

[13] M. Ali, I -N. Lin, C. -J. Yeh, Predictor Packing in Developing Unprecedented Shaped Colloidal Particles. Accepted in NANO (World Scientific)

[14] M. Ali, I -N. Lin, Phase transitions and critical phenomena of tiny grains carbon films synthesized in microwave-based vapor deposition system. http://arXiv.org/abs/1604.07152 (last version)

[15] M. Ali, M. Ürgen, Switching dynamics of morphology-structure in chemically deposited carbon films -A new insight, Carbon 122 (2017) 653-663.

[16] M. Ali, M. Ürgen, Deposition Chamber Pressure on the Morphology-Structure of Carbon Films. https://arxiv.org/abs/1802.00730 (last version)

[17] M. Ali, E. Hamzah, M. R. M. Toff, A. H. Hashim, The effect of Nitrogen gas flow rate on the properties of TiN-coated HSS using Cathodic Arc Evaporation PVD Technique, Surf. Rev. Lett. 12 (2005) 631-643.

[18] M. Ali, E. Hamzah, M. R. M. Toff, Effect of metal ion etching on the tribological, mechanical and microstructural properties of TiN-coated D2 tool steel using CAE PVD technique, Surf. Rev. Lett. 13 (2006) 413-421.

[19] M. Ali, E. Hamzah, M. R. M. Toff, Effect of substrate bias voltage on the microstructural and mechanical properties of TiN-coated HSS synthesized by CAE PVD technique, Surf. Rev. Lett. 13 (2006) 621-633.

[20] E. Hamzah, M. Ali, M. R. M. Toff, Effect of substrate bias on friction coefficient, adhesion strength and hardness of TiN-coated D2 tool steel deposited via cathodic arc PVD technique, Surf. Rev. Lett. 13 (2006) 763-771.

[21] M. Ali, E. Hamzah, M. R. M. Toff, Deposition and characterizations of TiN-coated steels at various $\mathrm{N}_{2}$ gas flow rates with constant etching by using cathodic arc physical vapour deposition technique, Surf. Rev. Lett. 14 (2007) 93-100. 
[22] E. Hamzah, A. Ourdjini, M. Ali, P. Akhter, M. R. M. Toff, M. A. Hamid, Influence of nitrogen flow rate on friction coefficient and surface roughness of TiN coatings deposited on tool steel using arc method, Surf. Rev. Lett. 14 (2007) 1007-1013.

[23] M. Ali, P. Akhter, E. Hamzah, M. R. M. Toff, I. A. Qazi, Effect of coating thickness on the properties of TiN coatings deposited on tool steels using cathodic arc PVD technique, Surf. Rev. Lett. 15 (2008) 401-410.

[24] M. Ali, E. Hamzah, M. R. M. Toff, Friction coefficient and surface roughness of TiN-coated HSS deposited using cathodic arc evaporation PVD technique, Ind. Lubr. Tribol. 60 (2008) 121-130.

[25] M. Ali, E. Hamzah, T. Abbas, M. R. M. Toff, 1. A. Qazi, Macrodroplet reduction and growth mechanisms in cathodic arc physical vapour deposition of TiN films, Surf. Rev. Lett. 15 (2008) 653-659.

[26] M. Ali, E. Hamzah, N. Ali, Adhesion strength of TiN coatings at various ion etching deposited on tool steels using cathodic arc PVD technique, Surf. Rev. Lett. 16 (2009) 29-35.

[27] M. Ali, E. Hamzah, I. A. Qazi, M. R. M. Toff, Effect of Cathodic Arc PVD Parameters on Roughness of TiN Coating on Steel Substrate, Curr. Appl. Phys. 10 (2010) 471-474.

[28] M. Ali, E. Hamzah, M. R. M. Toff, Study of macrodroplet and growth mechanisms with and without ion etchings on the properties of TiN coatings deposited on HSS using cathodic arc physical vapour deposition technique, Mater. Sci. Eng. A 474 (2008) 236-242.

[29] H. Elmkhah, F. Attarzadeh, A. F. -alhosseini, K. H. Kim. Microstructural and electrochemical comparison between TiN coatings deposited through HIPIMS and DCMS techniques, J. Alloy. Compd. 735 (2018) 422-429.

[30] J.C. Oliveiraa, F. Fernandesa, R. Serraa, A. Cavaleiro. On the role of the energetic species in TiN thin film growth by reactive deep oscillation magnetron sputtering in Ar/N2, Thin Solid Films 645 (2018) 253-264. 
[31] U. Vogel, S. Oswalda, J. Eckert. Interface and stability analysis of Tantalum- and Titanium nitride thin films onto Lithiumniobate, Appl. Surf. Sci. 425 (2017) 254260.

[32] G. T. P. Azar, C. Yelkarasi, M. Ürgen. The role of droplets on the cavitation erosion damage of TiN coatings produced with cathodic arc physical vapor deposition, Surf. Coat. Technol. 322 (2017) 211-217.

[33] A. Bahri, E. Kaçar, S.S. Akkaya, K. Elleuch, M. Ürgen. Wear protection potential of TiN coatings for 304 stainless steels used in rotating parts during olive oil extraction, Surf. Coat. Technol. 304 (2016) 560-566.

[34] M.F. Othman, A.R. Bushroa \& W.N.R. Abdullah. Evaluation techniques and improvements of adhesion strength for TiN coating in tool applications: a review, J. Adhes. Sci. Technol., 29 (2015) 569-591.

[35] Q.W. Jiang, G.R. Li, X.P. Gao, Highly ordered TiN nanotube arrays as counter electrodes for dye-sensitized solar cells, Chem. Commun. 44 (2009) 6720-6722.

[36] S.G. Harris, E.D. Doyle, Y.-C. Wong, P.R. Munroe, J.M. Cairney, J.M. Long. Reducing the macroparticle content of cathodic arc evaporated TiN coatings, Surf. Coat. Technol., 183 (2004) 283-294.

[37] L. von Fieandta, K. Johanssona, T. Larssonb, M. Bomana, E. Lindahlc. On the growth, orientation and hardness of chemical vapor deposited Ti(C,N), Thin Solid Films 645 (2018) 19-26.

[38] K. Bobzin, High-performance coatings for cutting tools, CIRP Jour. Manuf. Sci. Technol., 18 (2017) 1-9.

[39] Q. Wang, F. Zhou. Progress in Tribological Properties of Nano-Composite Hard Coatings under Water Lubrication, Lubricants 5 (2017) 5

[40] D. Music, R. W. Geyer, J. M. Schneider. Recent progress and new directions in density functional theory based design of hard coatings, Surf. Coat. Technol. 286 (2016) 178-190.

[41] E. Santecchiaa, A.M.S. Hamoudaa, F. Musharavatia, E. Zalnezhadb, M. Cabibboc, S. Spigarellic, Wear resistance investigation of titanium nitride-based coatings, Ceram. Int. 41 (2015) 10349-10379. 
[42] Y. X. Wang, S. Zhang. Toward hard yet tough ceramic coatings, Surf. Coat. Technol. 258 (2014) 1-16.

[43] A. Inspektor, P. A. Salvador. Architecture of PVD coatings for metalcutting applications: A review, Surf. Coat. Technol. 257 (2014) 138-153.

[44] A. D. Pogrebnjaka, A. V. Pshyka, V. M. Beresnevb, B. R. Zhollybekova. Protection of specimens against friction and wear using titanium-based multicomponent nanocomposite coatings: A review, J. Frict. Wear, 35 (2014) 5566.

[45] M. Fenker, M. Balzer, H. Kappl. Corrosion protection with hard coatings on steel: Past approaches and current research efforts, Surf. Coat. Technol., 257 (2014) 182-205.

[46] Y. Purandarea, A. Ehiasarian, A. Santana, P. Hovsepian. ZrN coatings deposited by high power impulse magnetron sputtering and cathodic arc techniques, J. Vac. Sci. Technol. A, 32 (2014) 031507.

[47] C. Mitterer, F. Holler, F. Üstel, D. Heim. Application of hard coatings in aluminium die casting -soldering, erosion and thermal fatigue behaviour, Surf. Coat. Technol., 125 (2000) 233-239.

[48] W.-D. Münz, T. Hurkmans, G. Keiren, T. Trinh. Comparison of TiAIN coatings grown by unbalanced magnetron and arc bond sputtering techniques, J. Vac. Sci. Technol. A, 11 (1993) 2583-2589.

[49] S.C. Glotzer, M.J. Solomon, Anisotropy of building blocks and their assembly into complex structures, Nature Mater. 6 (2007) 557-562.

[50] S. Link, M.A. El-Sayed, Shape and size dependence of radiative, nonradiative and photothermal properties of gold nanocrystals, Int. Rev. Phys. Chem. 19 (2000) 409- 453. 


\section{Authors' biography:}

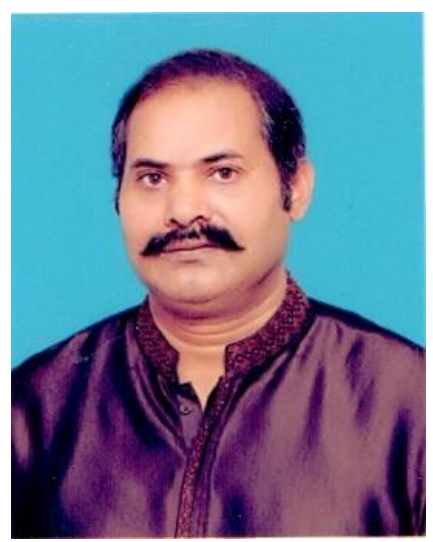

Mubarak Ali graduated from University of the Punjab with B.Sc. (Phys\& Maths) in 1996 and M.Sc. Materials Science with distinction at Bahauddin Zakariya University, Multan, Pakistan (1998); thesis work completed at Quaid-i-Azam University Islamabad. He gained Ph.D. in Mechanical Engineering from Universiti Teknologi Malaysia under the award of Malaysian Technical Cooperation Programme (MTCP;2004-07) and postdoc in advanced surface technologies at Istanbul Technical University under the foreign fellowship of The Scientific and Technological Research Council of Turkey (TÜBITAK; 2010). He completed another postdoc in the field of nanotechnology at Tamkang University Taipei (2013-2014) sponsored by National Science Council now M/o Science and Technology, Taiwan (R.O.C.). Presently, he is working as Assistant Professor on tenure track at COMSATS University Islamabad (previously known as COMSATS Institute of Information Technology), Islamabad, Pakistan (since May 2008) and prior to that worked as assistant director/deputy director at M/o Science \& Technology (Pakistan Council of Renewable Energy Technologies, Islamabad; 2000-2008). He was invited by Institute for Materials Research, Tohoku University, Japan to deliver scientific talk. He gave several scientific talks in various countries. His core area of research includes materials science, physics \& nanotechnology. He was also offered the merit scholarship for the PhD study by the Government of Pakistan, but he couldn't avail. $\mathrm{He}$ is author of several articles available at https://scholar.google.com.pk/citations?hl=en\&user=UYjvhDwAAAAJ, https://www.researchgate.net/profile/Mubarak Ali5.

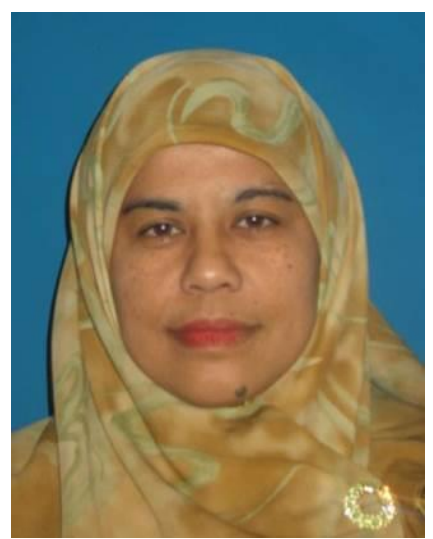

Dr Esah Hamzah is a senior standing Professor at Universiti Teknologi Malaysia in the Faculty of Mechanical Engineering. Dr Esah obtained her BSc degree from the University of Wales at Swansea, UK, and MSc and PhD in Metallurgy from the University of Manchester Institute of Science and Technology (U.M.I.S.T.) UK. She has over 30 years of experience in teaching the undergraduate and postgraduate courses related to metallurgy and materials engineering. She has also supervised both masters and PhD students and has served as an examiner for MSc and PhD theses. She has held various administrative positions at the Universiti Teknologi Malaysia and remained Head of Department (1999-2005) and Deputy Dean (Academics) of the Faculty of Mechanical Engineering (2005-2010). She has been actively involved in research namely in the areas of phase transformation and mechanical behavior of metals, metal failure, corrosion and coating. She has been awarded many research grants from the University and the Malaysian Government. She has also presented and published more than 200 papers in the national and international conferences and journals. Some of her papers won the "Best Paper" award at various International events. She is also a contributor to the University's Best Publication Award won by the Faculty of Mechanical Engineering in series of years along with Excellent Service for the year 1993 and the University Excellence Award in 2000, 2003 and 2007. Dr Esah is an active Council Member, charted engineer, and in pioneering Fellow members of the Institute of Materials Malaysia (IMM).

Dr. Mohd Radzi Mohd Toff is a General Manager at Advanced Materials Research Centre (AMREC), SIRIM BHD, Malaysia and prior to that worked as head of coating technology group at AMREC and principal scientist. He graduated from Universiti Kebangsaan Malaysian (UKM) and earned PhD in Chemical Engineering from The University of Sheffield, UK.

\section{Last and Final Version}

